Fernando Vinholes Siqueira ${ }^{1,11}$

Markus Vinícius Nahas

Luiz Augusto Facchini ${ }^{11, \mathrm{IV}}$

Denise Silva da Silveira"'I

Roberto Xavier Piccini"I

Elaine Tomasi"

Elaine Thumév

Felipe Fossati Reichert ${ }^{v}$

Pedro Rodrigues Curi Hallal ${ }^{\mathrm{VI}}$

Programa de Pós-Graduação em Educação Física. Universidade Federal de Santa Catarina. Florianópolis, SC, Brasil

Centro de Ciência da Vida e da Saúde. Universidade Católica de Pelotas. Pelotas, RS, Brasil

III Departamento de Medicina Social. Faculdade de Medicina. Universidade Federal de Pelotas (UFPel).

Pelotas, RS, Brasil

Iv Programa de Pós-Graduação em Epidemiologia. UFPel. Pelotas, RS, Brasil

Departamento de Educação Física. Universidade Estadual de Londrina. Londrina, PR, Brasil

Correspondência | Correspondence: Fernando Carlos Vinholes Siqueira Programa de Pós-Graduação em Educação Física

Universidade Federal de Santa Catarina

C.P. 476 Campus Universitário Trindade

88040-900 Florianópolis, SC, Brasil

E-mail: fcvsiqueira@uol.com.br

\section{Fatores considerados pela população como mais importantes para manutenção da saúde}

\author{
Factors considered important \\ for health maintenance by the \\ population
}

RESUMO

OBJETIVO: Analisar os fatores que adultos e idosos consideram como mais importantes para manutenção da saúde.

MÉTODOS: Estudo transversal realizado com 4.060 adultos e 4.003 idosos residentes em áreas de abrangência de 240 unidades básicas de saúde das regiões Sul e Nordeste, em 2005. Um cartão com figuras e frases referentes a sete fatores relacionados com o risco de doenças e agravos não transmissíveis era mostrado aos indivíduos para que indicassem o fator mais relevante para a saúde. Os fatores eram: manter uma alimentação saudável, fazer exercício físico regularmente, não tomar bebidas alcoólicas em excesso, realizar consultas médicas regularmente, não fumar, manter o peso ideal e controlar ou evitar o estresse. As análises foram ajustadas por regressão de Poisson com cálculo de razões de prevalência ajustadas, intervalos com $95 \%$ de confiança, e valores de significância usando os Testes de Wald para heterogeneidade e tendência linear.

RESULTADOS: Os fatores mais freqüentemente indicados pelos adultos foram: alimentação saudável $(33,8 \%)$, realizar exercício físico $(21,4 \%)$ e não fumar $(13,9 \%)$. Entre os idosos, os fatores mais relatados foram: alimentação saudável $(36,7 \%)$, não fumar $(17,7 \%)$ e consultar o médico regularmente $(14,2 \%)$. Foram observadas diferenças entre os fatores citados conforme a região geográfica, variáveis demográficas, socioeconômicas e de saúde.

CONCLUSÕES: A maioria de adultos e idosos, de ambas regiões, reconhece e indica a necessidade de manter uma alimentação saudável e de não fumar como medidas mais importantes para manutenção da saúde. Estratégias de educação em saúde devem considerar essas características dos indivíduos para estimular medidas específicas a serem adotadas para cada segmento populacional.

DESCRITORES: Adulto. Idoso. Conhecimentos, Atitudes e Prática em Saúde. Estilo de Vida. Fatores de Risco. Doença Crônica. Estudos Transversais. 


\section{ABSTRACT}

OBJECTIVE: To analyze factors that adults and elderly individuals regard as the most important for health maintenance.

METHODS: A cross-sectional study performed with 4,060 adults and 4,003 elderly individuals in areas covered by 240 primary health units in the Brazilian Southern and Northeastern regions, in 2005. A card with pictures and sentences about seven factors associated with the risk of non-communicable diseases and health problems was shown to individuals so they should point out the most relevant factor for health. These factors were as follows: to maintain a healthy diet, to exercise regularly, to avoid excessive drinking, to have regular medical check-ups, not to smoke, to maintain the ideal weight, and to control or avoid stress. Adjusted analysis was carried out by Poisson regression, with calculations of adjusted prevalence ratios, respective $95 \%$ confidence intervals and significance values, using Wald tests for heterogeneity and linear trend.

RESULTS: Factors most frequently indicated by adults were the following: to maintain a healthy diet $(33.8 \%)$, to exercise regularly $(21.4 \%)$ and not to smoke $(13.9 \%)$. Among the elderly, factors most frequently reported were: to maintain a healthy diet (36.7\%), not to smoke $(17.7 \%)$ and to have regular medical check-ups (14.2\%). Differences among factors mentioned were observed, according to geographical region, and demographic, socioeconomic and health variables.

CONCLUSIONS: The majority of adults and elderly individuals of both regions recognize and indicate the need to maintain a healthy diet and not to smoke as the most important health maintenance measures. Health education strategies should consider these characteristics to promote specific measures to be adopted for each population segment.

DESCRIPTORS: Adult. Aged. Health Knowledge, Attitudes, Practice. Life Style. Risk Factors. Chronic Disease. Cross-Sectional Studies.

\section{INTRODUÇÃO}

A Declaração de Alma-Ata, ${ }_{11}^{11}$ que concita a ação internacional para o desenvolvimento de estratégias direcionadas a melhorar os cuidados relacionados à saúde dos povos, está completando 30 anos. Na Declaração, a educação em saúde é incluída no item relacionado aos cuidados primários à saúde como elemento fundamental na busca da prevenção e controle de doenças e promoção da saúde. ${ }^{11}$ No Brasil, a Política Nacional da Atenção Básica estabelece que se busquem ações em saúde que abranjam a responsabilidade em todas as esferas da gestão, de viabilizar a capacitação e educação permanente em saúde. ${ }^{6}$

Nesta perspectiva, a atenção básica é importante ao representar para a população o aconselhamento visando à adoção de hábitos de vida saudáveis. Entre os aconselhamentos, os relacionados à prática de atividade física (AF) têm sido incluídos em várias estratégias globais em saúde. Da mesma forma, são ressaltadas orientações nutricionais, abandono de hábitos prejudiciais como o tabagismo e o alcoolismo, cuidados relacionados à obesidade e acesso aos serviços de saúde., ${ }^{2,7}$
Embora o conhecimento sobre fatores de risco e proteção à saúde seja bem disseminado, pouco se sabe sobre a percepção populacional sobre a importância dessas exposições, bem como o grau de importância dado a esses fatores.

O objetivo do presente estudo foi analisar os fatores que adultos e idosos consideram como mais importantes para manutenção da saúde.

\section{MÉTODOS}

Estudo transversal realizado com amostra de indivíduos adultos (30 a 64 anos) e idosos (65 anos ou mais), residentes nas áreas de abrangência das unidades básicas de saúde (UBS) de 41 municípios com mais de 100 mil habitantes das regiões Sul e Nordeste, assim distribuídos: 17 no Rio Grande do Sul, quatro em Santa Catarina (Sul), dois em Alagoas, três na Paraíba, dez em Pernambuco, dois no Piauí e três no Rio Grande do Norte (Nordeste), incluindo todas as capitais dos 
estados estudados, no período entre março e agosto de 2005. A pesquisa integrou o Estudo de Linha de Base do Projeto de Expansão e Consolidação da Saúde da Família (PROESF), cujos detalhes metodológicos estão disponíveis em outras publicações. ${ }^{5,6,13}$

De posse de listas produzidas pelas secretarias de saúde dos municípios, foram selecionadas aleatoriamente 120 UBS com duas modalidades de atenção básica: Programa Saúde da Família (PSF) e modelo tradicional. As unidades de PSF possuíam equipes de saúde da família, com médico generalista, enfermeiro, auxiliares de enfermagem e agentes comunitários. As unidades tradicionais caracterizavam-se pela presença na equipe de médicos especialistas (clínico, pediatra e ginecoobstetra), enfermeiro, auxiliar de enfermagem e pessoal administrativo para apoio técnico, contando ainda, em caráter eventual, com o apoio de outras especialidades profissionais (dentista, nutricionista, fisioterapeuta) e mesmo, de outras especialidades médicas. Na seleção, foi estabelecida uma proporcionalidade com o tamanho da rede básica dos municípios: municípios com rede maior contribuíram com maior número de unidades. Na região Sul, obteve-se uma amostra de 69 UBS de PSF e 51 tradicionais. No Nordeste, a amostra foi constituída de 79 UBS de PSF e 41 tradicionais.

Adultos e idosos foram selecionados de forma aleatória sistemática entre aqueles residentes na área de abrangência das UBS. Com base na obtenção prévia do mapa da área de abrangência das UBS, uma estimativa populacional foi realizada a partir das áreas censitárias do Instituto Brasileiro de Geografia e Estatística (IBGE). Nos domicílios sorteados, apenas um morador era selecionado para participar do estudo. Os questionários foram aplicados por 15 supervisores treinados.

As variáveis dependentes do estudo foram construídas com base em um cartão mostrado aos indivíduos contendo frases e figuras relacionadas a sete fatores associados à manutenção da saúde. Os fatores eram: manter uma alimentação saudável, fazer exercício físico regularmente, não tomar bebidas alcoólicas em excesso, realizar consultas médicas regularmente, não fumar, manter o peso ideal e controlar ou evitar o estresse. Os entrevistados deveriam indicar a medida que eles consideravam a mais importante para a manutenção da sua saúde.

As seguintes variáveis independentes foram incluídas em modelo de análise hierarquizado: sexo, idade, cor da pele, padrão de consumo socioeconômico ${ }^{\mathrm{a}}$ e escolaridade no primeiro nível; situação conjugal, tabagismo, nível insuficiente de atividade física $(<150$ minutos por semana de prática de AF) no segundo nível; e diagnóstico médico de hipertensão arterial sistêmica, diagnóstico médico de diabetes, diagnóstico médico de doença dos nervos, autopercepção de saúde, trabalho remunerado no último mês no terceiro nível.

As análises descritivas incluíram cálculos de proporções e respectivos intervalos com $95 \%$ de confiança. A prevalência dos desfechos foi calculada para o grupo das variáveis independentes. Também foram verificadas possíveis diferenças nos desfechos em relação à região (Sul e Nordeste). A análise ajustada foi realizada por regressão de Poisson com cálculo de razões de prevalência ajustadas, intervalos com 95\% de confiança, e valores de significância usando os Testes de Wald para heterogeneidade e tendência linear. ${ }^{1}$ Todas as análises levaram em consideração o desenho amostral, e um modelo hierárquico de determinação do desfecho. ${ }^{1}$ Variáveis com valor de $\mathrm{p} \leq 0,20$ foram mantidas no modelo de análise como estratégia para controle de possível confusão. ${ }^{9}$ As análises foram realizadas no pacote estatístico Stata 9.2.

Um total de 4.060 adultos e 4.003 idosos foi entrevistado. A taxa de perdas foi de $3,4 \%$ entre os adultos e $4,7 \%$ entre os idosos.

O estudo foi aprovado pelo Comitê de Ética da Faculdade de Medicina da Universidade Federal de Pelotas. Foi obtido consentimento esclarecido de todos os participantes.

\section{RESULTADOS}

As Tabelas 1 e 2 descrevem a amostra de adultos e idosos separadamente em relação às variáveis independentes do estudo. O percentual de mulheres foi superior entre os idosos $(61,2 \%)$ do que entre os adultos $(55,1 \%)$. A cor da pele predominante foi branca em ambos os grupos etários. Entre os idosos, tanto o padrão de consumo socioeconômico quanto a escolaridade foram mais baixos do que entre os adultos. Entre os adultos, a maioria era casada ou vivia com companheiro $(73,3 \%)$, enquanto entre os idosos a maior prevalência foi de viúvos (45,0\%). O tabagismo foi citado por $27,7 \%$ dos adultos, enquanto entre os idosos foi de 15,2\%. Em relação ao nível de AF, 31,8\% dos adultos não alcançaram os escores mínimos recomendados (pelo menos 150 minutos por semana de AF moderadas), enquanto entre os idosos a prevalência foi de 58,0\%. Em relação a diagnóstico referido de hipertensão arterial, a prevalência entre os idosos $(63,5 \%)$ foi mais de duas vezes a dos adultos (28,0\%). A mesma relação se apresentou no caso de diabetes, porém com uma magnitude menor: $6,7 \%$ entre adultos e $19,5 \%$ entre os idosos. Para doença dos nervos, o relato positivo foi semelhante para os grupos de adultos e idosos (Tabela 1).

a Associação Brasileira de Empresas de Pesquisa. Critério de classificação econômica do Brasil. São Paulo: Associação Brasileira de Empresas de Pesquisa; 2002 [citado 2009 out 19]. Disponível em: http://www.abep.org/?usaritem=arquivos\&iditem=23 
Tabela 1. Características sociodemográficas da amostra de adultos e idosos residentes em áreas de abrangência de unidades básicas de saúde. Regiões Sul e Nordeste do Brasil, 2005.

\begin{tabular}{|c|c|c|c|c|}
\hline Variável & Adultos (\%) & $n$ & Idosos $(\%)$ & $\mathrm{n}$ \\
\hline Sexo & & 4060 & & 4003 \\
\hline Masculino & 44,9 & 1822 & 38,8 & 1554 \\
\hline Feminino & 55,1 & 2238 & 61,2 & 2449 \\
\hline Idade (anos) & & 4059 & & 4003 \\
\hline 30 a 40 & 36,2 & 1470 & - & \\
\hline 41 a 50 & 31,1 & 1264 & - & \\
\hline 51 a 64 & 32,7 & 1325 & - & \\
\hline 65 a 70 & - & & 38,5 & 1542 \\
\hline 71 a 75 & - & & 25,7 & 1030 \\
\hline 76 a 79 & - & & 18,5 & 739 \\
\hline 80 ou mais & - & & 17,3 & 692 \\
\hline Cor da pele & & 4039 & & 3985 \\
\hline Branca & 65,3 & 2636 & 70,0 & 2789 \\
\hline Não branca & 34,7 & 1403 & 30,0 & 1196 \\
\hline Padrão de consumo socioeconômico & & 3908 & & 3650 \\
\hline A, B ou C & 37,4 & 1463 & 21,0 & 766 \\
\hline $\mathrm{D}$ & 32,4 & 1267 & 33,7 & 1231 \\
\hline $\mathrm{E}$ & 30,2 & 1178 & 45,3 & 1653 \\
\hline Escolaridade ${ }^{a}$ & & 4047 & & 3923 \\
\hline Nenhuma & 16,7 & 675 & 49,4 & 1938 \\
\hline Fundamental incompleto & 47,6 & 1927 & 43,2 & 1694 \\
\hline Fundamental completo & 11,5 & 464 & 4,0 & 155 \\
\hline Médio incompleto & 4,9 & 200 & - & \\
\hline Médio completo & 14,9 & 602 & - & \\
\hline Superior & 4,4 & 179 & 3,4 & 136 \\
\hline Situação conjugal & & 4060 & & 3970 \\
\hline Casado ou vive com companheiro(a) & 73,3 & 2975 & 42,7 & 1694 \\
\hline Viúvo(a) & 7,1 & 287 & 45,0 & 1785 \\
\hline Separado(a) divorciado(a) & 9,8 & 400 & 7,8 & 310 \\
\hline Solteiro(a) & 9,8 & 398 & 4,5 & 181 \\
\hline Trabalho remunerado no último mês & & 4056 & & 3957 \\
\hline Não & 51,3 & 2081 & 94,7 & 3747 \\
\hline Sim & 48,7 & 1975 & 5,3 & 210 \\
\hline
\end{tabular}

a Para idosos a última categoria refere-se ao grupo de $2^{\circ}$ grau completo e superior

As prevalências das medidas mais importantes para manter uma boa saúde, indicadas pelos adultos, foram: manter uma alimentação saudável (33,8\%; IC 95\%: $32,3 ; 35,2)$; realizar exercício físico regularmente (21,4\%; IC 95\%: 20,2;22,7); não fumar (13,9\%; IC 95\%: $12,9 ; 15,0)$; consultar médico regularmente $(13,6 \%$; IC $95 \%$ : 12,$5 ; 14,7)$; não ingerir álcool $(6,4 \%$; IC 95\%: $5,6 ; 7,1)$; evitar o estresse $(6,2 \%$; IC 95\%: 5,5;7,0); manter o peso ideal (4,6\%; IC 95\%: 4,0;5,3).

No Sul, as prevalências para os mesmos desfechos foram de 34,9\% para alimentação, 20,6\% para exercício físico, $15,0 \%$ para não fumar, $11,7 \%$ para consulta ao médico, mantendo até este ponto a mesma tendência da amostra geral. O item a seguir mais citado no Sul foi evitar o estresse $(6,8 \%)$, seguido da medida para evitar o álcool $(5,9 \%)$ e manter o peso ideal $(5,1 \%)$. No Nordeste, $32,8 \%$ dos indivíduos indicaram primeiramente manter uma boa alimentação, 22,2\% realizar exercício físico, $15,3 \%$ visitar o médico regularmente, $13,0 \%$ não fumar, 6,8\% não ingerir álcool, 5,7\% não estresse e $4,2 \%$ manter o peso ideal.

As Tabelas 3 e 4 mostram as prevalências e a análise ajustada do desfecho entre os adultos residentes em áreas de abrangência de UBS. A indicação da medida 
Tabela 2. Características comportamentais e de saúde da amostra de adultos e idosos residentes em áreas de abrangência de unidades básicas de saúde. Regiões Sul e Nordeste do Brasil, 2005.

\begin{tabular}{|c|c|c|c|c|}
\hline Variável & Adultos (\%) & $\mathrm{n}$ & Idosos (\%) & $\mathrm{n}$ \\
\hline Tabagismo & & 4060 & & 4000 \\
\hline Não & 72,3 & 1864 & 84,8 & 3393 \\
\hline Sim & 27,7 & 2196 & 15,2 & 607 \\
\hline Sedentarismo & & 4023 & & 3944 \\
\hline Não & 68,2 & 2744 & 42,0 & 1658 \\
\hline Sim & 31,8 & 1279 & 58,0 & 2286 \\
\hline Diagnóstico médico de hipertensão & & 3960 & & 3963 \\
\hline Não & 72,0 & 2850 & 36,5 & 1447 \\
\hline Sim & 28,0 & 1110 & 63,5 & 2156 \\
\hline Diagnóstico médico de diabetes mellitus & & 3897 & & 3909 \\
\hline Não & 93,3 & 3634 & 80,5 & 3147 \\
\hline Sim & 6,7 & 263 & 19,5 & 762 \\
\hline Diagnóstico médico de doença dos nervos & & 4033 & & 3956 \\
\hline Não & 74,0 & 2985 & 71,0 & 2808 \\
\hline Sim & 26,0 & 1048 & 29,0 & 1148 \\
\hline Autopercepção da saúde & & 4048 & & 3951 \\
\hline Excelente & 6,7 & 272 & 2,6 & 104 \\
\hline Muito boa & 6,3 & 254 & 3,2 & 125 \\
\hline Boa & 38,3 & 1550 & 29,7 & 1174 \\
\hline Regular & 38,2 & 1548 & 43,8 & 1732 \\
\hline Ruim & 10,5 & 424 & 20,7 & 816 \\
\hline
\end{tabular}

alimentação saudável esteve associada com o sexo feminino, padrão de consumo socioeconômico A, B e $\mathrm{C}$ e não tabagismo. Para a indicação da medida fazer exercício físico regularmente, houve associação com sexo masculino, idade entre 30 e 40 anos, escolaridade superior e não diagnóstico de doença dos nervos. A única variável que se associou a não ingerir álcool foi ser de nível socioeconômico E. Consultar o médico regularmente mostrou-se associada ao sexo feminino, à cor de pele não branca e não ter trabalhado com remuneração no último mês. $\mathrm{O}$ desfecho não fumar esteve associado com menor escolaridade, tabagismo, ser fisicamente ativo e não diagnóstico médico de diabetes. A medida manter o peso ideal esteve associada ao sexo feminino e ao diagnóstico de hipertensão. Por fim, a indicação da necessidade de evitar o estresse foi mais freqüente entre mulheres, de cor de pele branca, não diabéticas e com doença dos nervos.

Entre os idosos, as medidas mais citadas como importantes para manter uma boa saúde foram: manter uma alimentação saudável (36,7\%; IC 95\%: 35,2;38,3); não fumar $(17,7 \%$; IC 95\%: 16,5;18,9); consultar médico regularmente $(14,2 \%$; IC 95\%: 13,0;15,2); fazer exercício físico (13,2\%; IC 95\%: 12,1;14,2); não ingerir álcool (8,1\%; IC 95\%: 7,3;9,0); evitar o estresse $(6,2 \%$; IC 95\%: 5,$5 ; 7,0)$; manter o peso ideal $(6,1 \%$; IC $95 \%: 5,3 ; 6,9)$.
No Sul, as prevalências para os mesmos desfechos foram de 38,5\% para alimentação, 17,5\% para não fumar, $13,3 \%$ para consulta ao médico, $13,1 \%$ para AF. O item a seguir mais citado no Sul foi evitar o álcool $(8,0 \%)$, seguido por evitar o estresse $(6,1 \%)$, e finalmente manter o peso ideal $(3,5 \%)$. No Nordeste, $35,1 \%$ dos indivíduos indicaram primeiramente manter uma boa alimentação, 17,9\% não fumar, 14,9\% visitar o médico regularmente, $13,1 \%$ realizar exercício físico, $8,3 \%$ não ingerir álcool, 6,1\% evitar estresse e 4,5\% manter o peso ideal.

As Tabelas 5 e 6 mostram as prevalências e análises ajustadas para as variáveis estudadas em relação ao desfecho entre idosos. Em relação à alimentação saudável, houve associação com sexo feminino, não tabagismo e ausência de doença de nervos. Relatar a medida fazer exercício físico esteve associado com idade de 65 a 70 anos e ser fisicamente ativo. A medida não ingerir álcool associou-se ao sexo masculino, ser fisicamente ativo, ter doença dos nervos e ter trabalho remunerado no último mês. Para o desfecho consultar o médico regularmente, houve associação apenas com sedentarismo, enquanto para não fumar, foi observada associação com sexo masculino, ausência de escolaridade e tabagismo. Manter o peso ideal esteve associado com o sexo feminino e evitar o estresse com tabagismo e diagnóstico médico de doença dos nervos. 


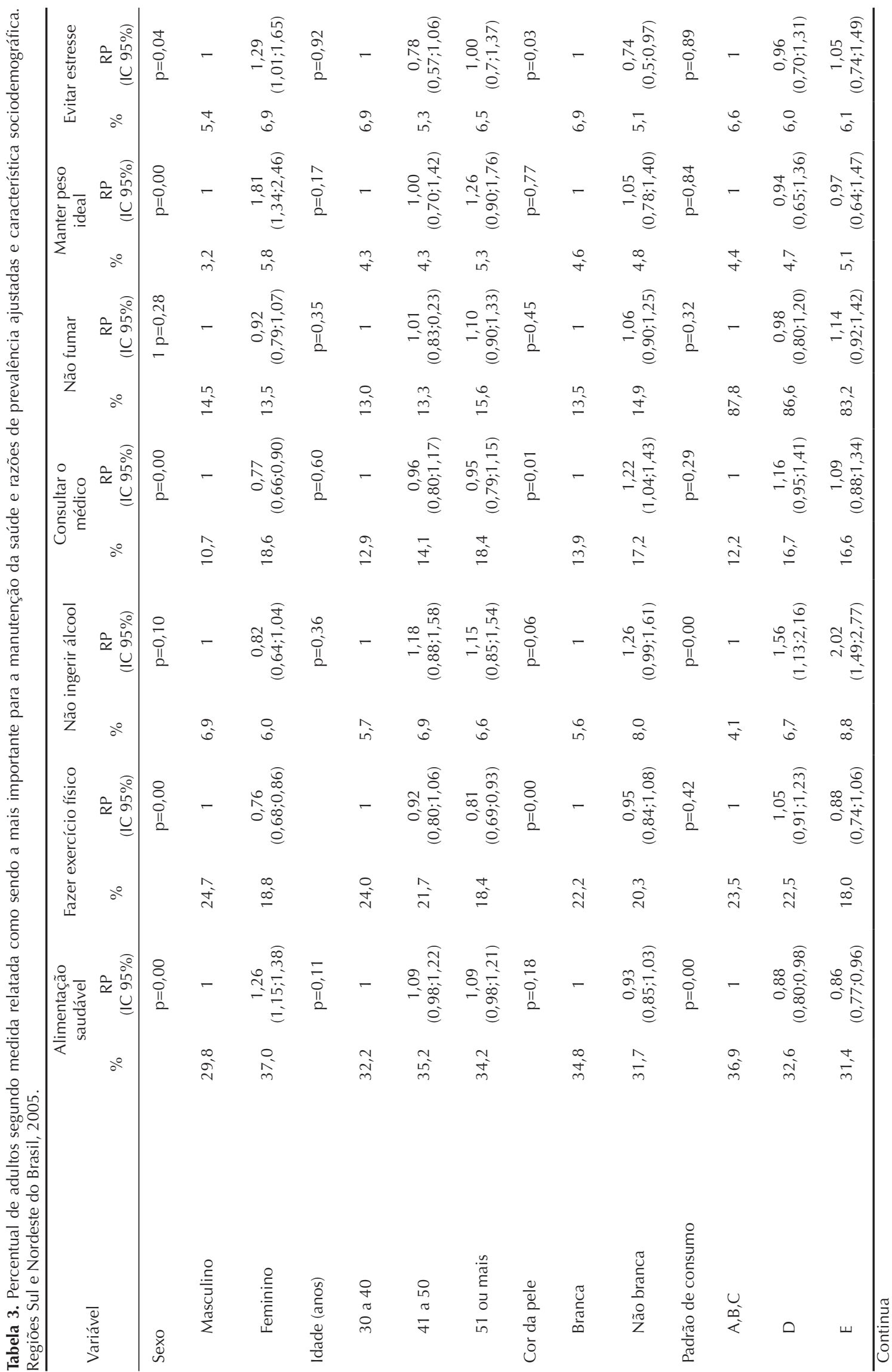




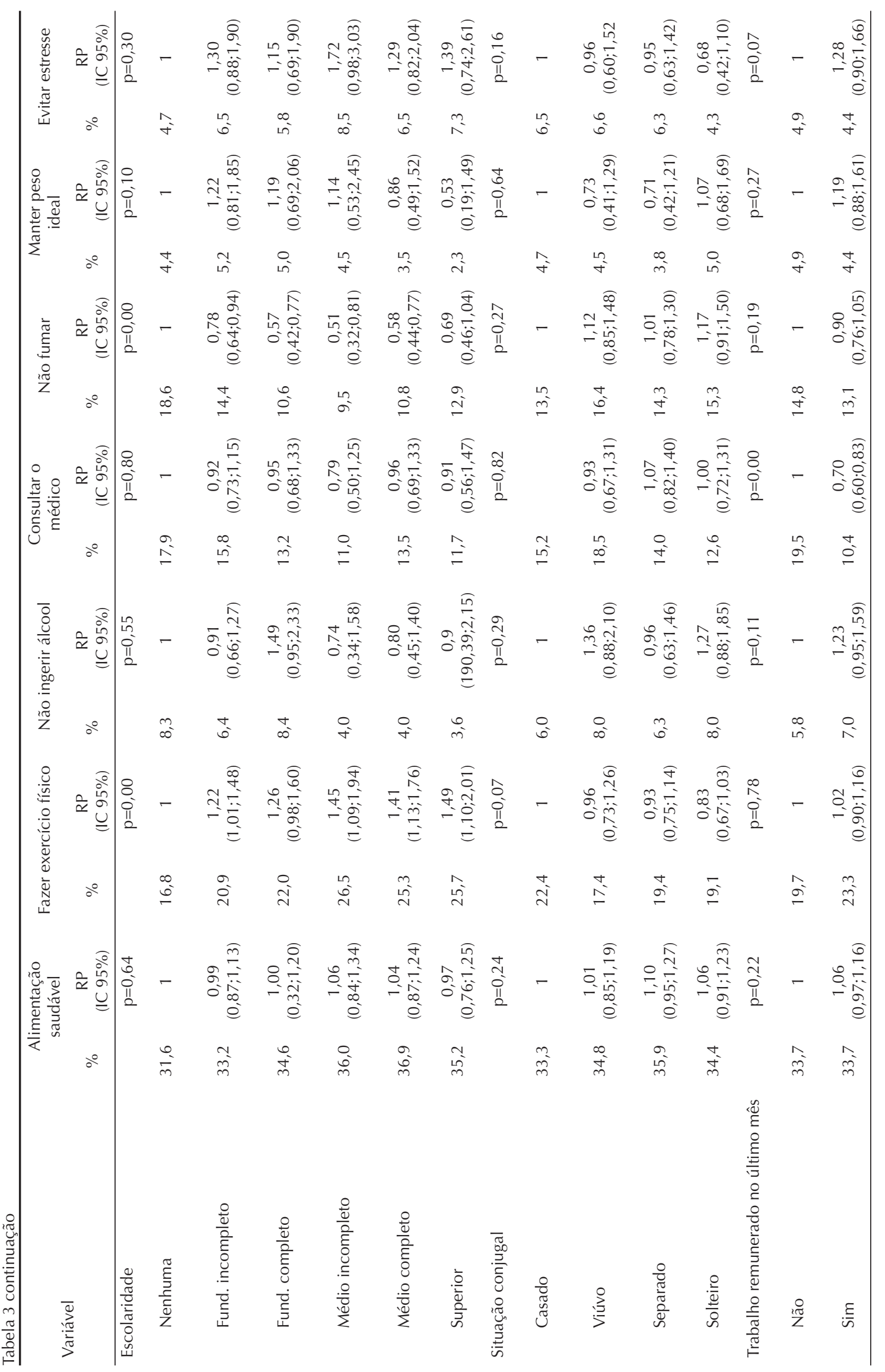


Entre os adultos e idosos, o sexo foi uma variável importante na indicação de diversas medidas, estando associada a cinco das sete medidas entre adultos (alimentação saudável [feminino, $\mathrm{p}<0,001$ ], fazer exercício físico [masculino, $\mathrm{p}<0,001$ ], consultar o médico [feminino, $p<0,001$ ], manter o peso ideal [feminino, $\mathrm{p}<0,001$ ] e evitar o estresse [feminino, $\mathrm{p}=0,04]$ ) e a quatro entre os idosos (alimentação saudável [feminino, $\mathrm{p}<0,001$ ], não ingerir álcool [masculino, $\mathrm{p}=0,01$ ], não fumar [masculino, $\mathrm{p}=0,002]$ e manter o peso ideal [feminino, $\mathrm{p}=0,01]$ ). A idade foi determinante somente para a indicação de fazer exercício físico regularmente nos grupos estudados, (adultos 30 a 40 anos, $\mathrm{p}=0,003$ e idosos 65 a 70 anos, $p=0,02$ ). A cor da pele esteve associada à indicação de duas medidas entre os adultos (consultar o médico regularmente [não branca, $\mathrm{p}=0,006$ ] e evitar o estresse [branca, $\mathrm{p}=0,03$ ]) e nenhuma entre os idosos. A escolaridade coincidiu na indicação de não fumar entre os dois grupos estudados (adultos, $\mathrm{p}<0,001$ e idosos $p=0,000$ ) e foi determinante para a indicação de fazer exercício físico entre os adultos (nível superior, $\mathrm{p}=0,002$ ). Entre adultos e idosos, os não tabagistas indicaram alimentação saudável como importante (adultos, $\mathrm{p}=0,02$ e idosos, $\mathrm{P}=0,004)$, e os tabagistas, não fumar (adultos, $\mathrm{p}<0,001$ e idosos, $\mathrm{p}=0,000$ ). Somente no grupo de idosos, os tabagistas indicaram a necessidade de evitar o estresse $(\mathrm{p}=0,009)$.

O nível insuficiente de atividade física $(<150 \mathrm{~min} / \mathrm{sem})$ foi uma variável fortemente associada à identificação de medidas de saúde entre os idosos, dos quais os suficientemente ativos indicaram fazer exercício físico $(p=0,02)$ e não ingerir álcool $(p=0,02)$. Ainda entre os idosos, os insuficientemente ativos indicaram a medida consultar o médico regularmente $(\mathrm{p}=0,02)$. Entre os adultos, somente não fumar $(\mathrm{p}=0,03)$ foi indicada mais freqüentemente pelos ativos fisicamente. Em relação ao diagnóstico de doenças crônicas como hipertensão e diabetes, somente no grupo de adultos, foram verificadas associações com, respectivamente, a necessidade de manter o peso ideal $(\mathrm{p}=0,002)$ e a importância de não fumar ( $\mathrm{p}=0,03)$. Os que não tinham diabetes indicaram a necessidade de evitar o estresse $(\mathrm{p}=0,05)$.

Para aqueles com diagnóstico de doença dos nervos, entre os adultos, houve associação com fazer exercício físico $(\mathrm{p}<0,001)$ e evitar o estresse $(\mathrm{p}<0,001)$. Nos idosos, a associação foi com alimentação saudável $(\mathrm{p}=0,008)$ para os que não possuíam diagnóstico, e com não ingerir álcool $(\mathrm{p}=0,04)$ e evitar o estresse $(\mathrm{p}=0,000)$, para os que possuíam tal diagnóstico. Em relação à variável percepção de saúde, somente nos idosos houve associação com a indicação de alimentação saudável $(\mathrm{p}=0,05)$ naqueles que percebiam sua saúde como muito boa. Consultar o médico regularmente esteve associado $(p<0,001)$ para os adultos que não trabalharam no último mês e não ingerir álcool esteve associado $(p=0,04)$ com idosos que trabalharam com remuneração no último mês.

\section{DISCUSSÃO}

Este é o primeiro estudo a investigar os fatores considerados mais importantes para a manutenção da saúde, por adultos e idosos, tanto na metodologia, nos desfechos investigados, e na abrangência em relação as duas regiões. O presente estudo revela que a maioria de adultos e idosos reconhece e indica a alimentação como uma fundamental medida para manter uma vida saudável. Esta escolha foi consistente nas duas regiões e grupos etários investigados. Segundo Dittrich et al (1995), as estruturas de cuidados em saúde devem ser organizadas no sentido de uma colaboração entre a prática médica e a consulta nutritiva para os pacientes. ${ }^{4}$ Nos Estados Unidos, estudo realizado com estudantes de medicina mostra que o aconselhamento nutricional pode melhorar o comportamento dos pacientes em relação à dieta. ${ }^{14} \mathrm{O}$ resultado encontrado provavelmente manifesta a preocupação, já existente, entre adultos e idosos, em relação à necessidade de manter uma boa alimentação como uma estratégia de manutenção de uma vida saudável.

Outro resultado consistente entre Sul e Nordeste foi a indicação de não fumar. A intensificação da divulgação e dos aconselhamentos em relação aos problemas causados pelo tabagismo mostra algum resultado positivo, conforme identificado pelos resultados do presente estudo. Monteiro et al ${ }^{10}$ (2007) creditam a diminuição substancial no hábito de fumar entre os adultos em torno de 35\% entre 1989 e 2003 ao aumento da divulgação de mensagens contra o tabagismo e a programas de prevenção do início do tabagismo entre os jovens.

Da mesma forma, consultar o médico regularmente obteve terceiro lugar entre as prevalências prioritárias dos indivíduos da amostra. Uma explicação pode ser o fato de a nossa amostra ser composta de indivíduos que residem em áreas de UBS, com possibilidade de um maior aconselhamento por parte dos profissionais dos postos de saúde para as pessoas consultarem o médico regularmente. Ainda, a população estudada, por residir em área de abragência de UBS, é mais pobre do que a população em geral, necessitando de mais cuidados em saúde. ${ }^{8}$

O estudo de Dias da Costa $^{3}$ (2008) mostra que as pessoas com sobrepeso e obesidade consultam o médico mais freqüentemente, o que poderia explicar a indicação da necessidade de manter o peso ideal pelos idosos, devido a maior exposição deste grupo a aconselhamento durante as consultas. O mesmo estudo mostrou também que pessoas adultas dão grande importância a consultar o médico, a exemplo de os adultos de 50 anos ou mais consultarem o médico com freqüência três vezes maior do que a dos mais jovens.

O exercício físico foi a quarta prioridade mais prevalente. No Brasil, ainda é escassa tal orientação como 


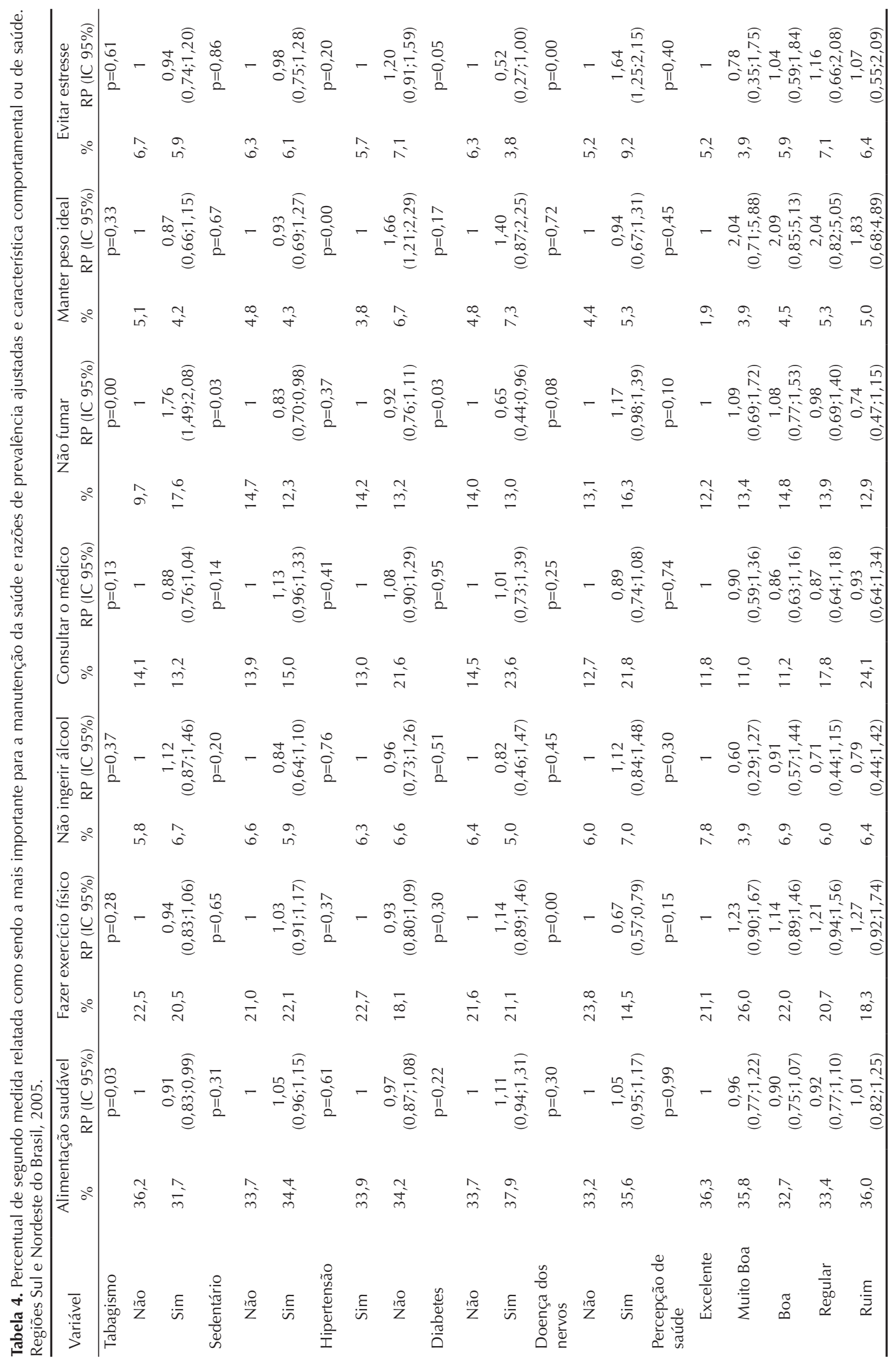




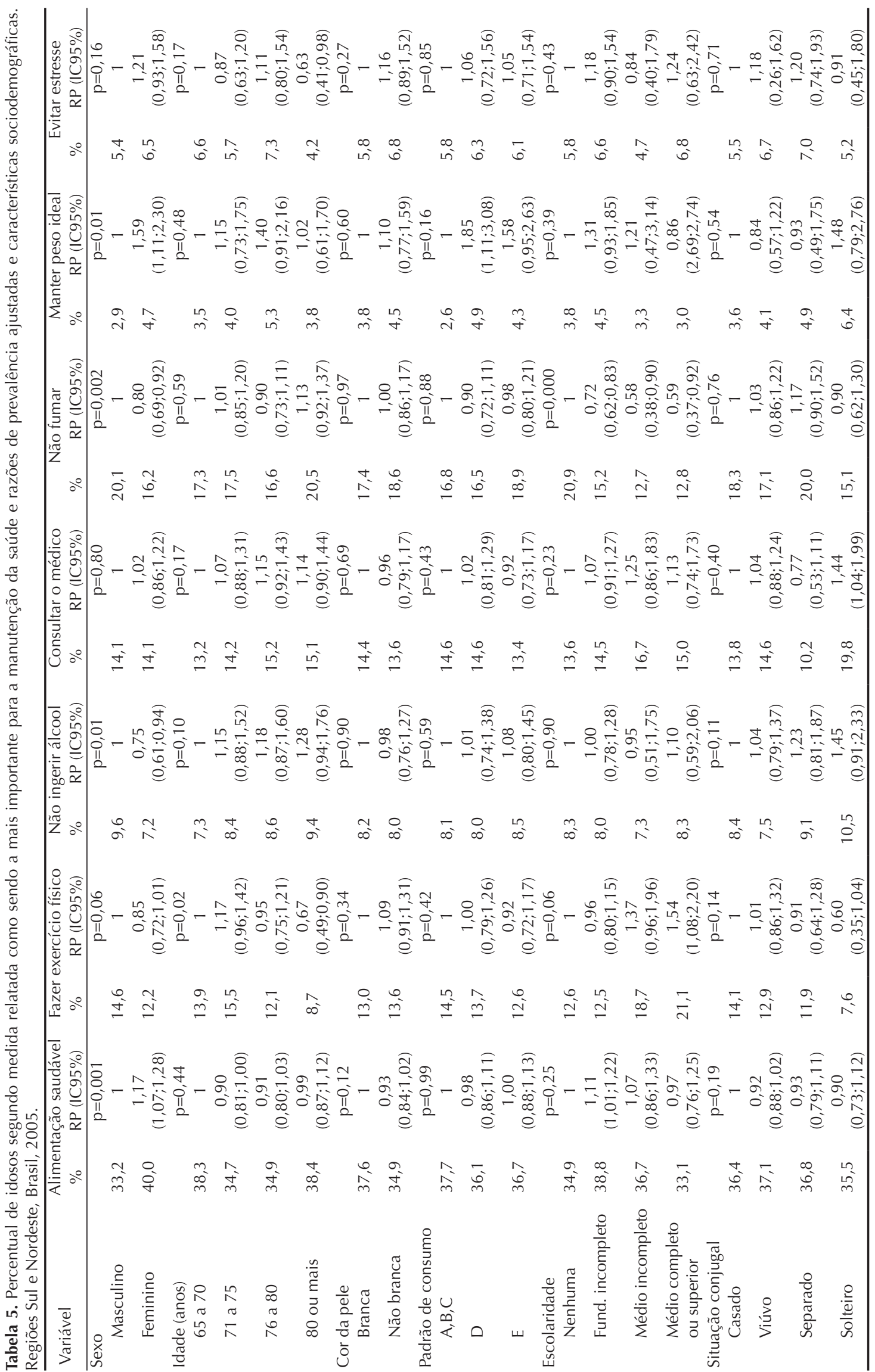


estratégia de educação em saúde na rede básica; logo, é de se esperar que esta medida não seja muito lembrada como prioridade em saúde..$^{2,7} \mathrm{O}$ estudo de Siqueira et a ${ }^{13}$ (2008) mostrou que, entre os adultos, somente 23,9\% receberam orientação para a prática de atividade física na UBS no último ano, enquanto o mesmo valor para os idosos foi de 30,3\%. Estudos recomendam a AF como estratégia para uma vida saudável frente a diversas morbidades, o que reforça a necessidade de se aumentarem o aconselhamento e a prescrição de AF. ${ }^{12}$

As prevalências das medidas priorizadas, estratificadas pela idade, mostram algumas diferenças em relação aos achados para a amostra total. Entre os adultos, a medida mais prevalente sempre foi manter uma alimentação saudável e a segunda, diferentemente da amostra total do estudo, foi fazer exercício físico regularmente. A terceira medida priorizada pelos adultos é a mesma da amostra total e do Sul: não fumar; no Nordeste, a terceira medida foi consultar o médico regularmente. Com relação à diferença entre a priorização das medidas fazer exercício físico nos adultos e consultar o médico regularmente nos idosos, acreditamos estar relacionada à exposição ao conhecimento sobre os fatores: enquanto em alguns locais predominam os anúncios para a prática regular de exercício físico, em outros locais ainda existe uma predominância dos anúncios para a procura regular de médicos. ${ }^{3}$

Entre os idosos, o mesmo resultado encontrado para a amostra total foi encontrado no Sul e no Nordeste. A principal diferença em relação às medidas priorizadas pelos adultos foi a escolha de consultar o médico em detrimento de realizar exercício físico regularmente. Como a amostra do estudo está composta por população mais pobre, é possível que estes fatores influenciem essa diferença. O PSF tem uma história mais longa no Nordeste, contando com a presença dos agentes comunitários de saúde, o que pode ter influenciado a população, de abrangência da UBS.

As orientações em saúde devem ser melhoradas no sentido de estimular mudanças de comportamento dos indivíduos. São necessárias ações coordenadas entre gestores e os diferentes profissionais de saúde que prestam seus serviços em UBS, bem como a qualificação do quadro de profissionais no sentido de melhorar as orientações em áreas pouco lembradas.

\section{REFERÊNCIAS}

1. Barros AJ, Hirakata VN. Alternatives for logistic regression in cross-sectional studies: an empirical comparison of models that directly estimate the prevalence ratio. BMC Med Res Methodol. 2003;3:21. DOI:10.1186/1471-2288-3-21

2. Chakravarthy MV, Joyner MJ, Booth FW. An obligation for primary care physicians to prescribe physical activity to sedentary patients to reduce the risk of chronic health conditions. Mayo Clin Proc. 2002;77(2):165-73. DOI:10.4065/77.2.165

3. Dias da Costa JS, Olinto MT, Gigante DP, Menezes AMB, Macedo S, Daltoé T,et al. Utilização de serviços ambulatoriais de saúde em Pelotas, Rio Grande do Sul, Brasil: alguns fatores relacionados com as consultas médicas acima da média. Cad Saude Publica. 2008;24(2)353-63. DOI:10.1590/S0102311X2008000200014

4. Dittrich A, Friebe D, Zunft HJ, Leonhauser IU. Health promotion and nutrition counseling for obese patients-a responsibility of medical care. Z Arztl Fortbild (Jena). 1995;89(4):382-5.

5. Facchini LA, Piccini RX, Tomasi E, Thumé E, Teixeira VA, Silveira DS, et al. Avaliação de efetividade da atenção básica à saúde em municípios das regiões Sul e Nordeste do Brasil: contribuições metodológicas. Cad Saude Publica. 2008;24(Supl 1):159-72. DOI:10.1590/S0102-311X2008001300020

6. Facchini LA, Piccini RX, Tomasi E, Thumé E, Silveira DS, Siqueira FV, et al. Desempenho do PSF no Sul e no Nordeste do Brasil: avaliação institucional e epidemiológica da Atenção Básica a Saúde. Cienc Saude Coletiva. 2006;11(3)669-81. DOI:10.1590/ S1413-81232006000300015

7. Fuscaldo JM. Prescribing physical activity in primary care. WV Med J. 2002;98(6):250-3.

8. Hart JT. The inverse care law. Lancet. 1971;1(7696):405-12.

9. Maldonado G, Greenland S. Simulation study of confounder-selection strategies. Am / Epidemiol. 1993;138(11):923-36.

10. Monteiro CA, Cavalcante TM, Moura EC, Claro RM, Szwarcwald CL. Population-based evidence of a strong decline in the prevalence of smokers in Braszil (19892003). Bull World Health Organ. 2007;85(7):527-34. DOI:10.2471/BLT.06.039073

11. Organización Mundial de la Salud. Conferencia Internacional sobre Atención Primaria de Salud. Atención primaria de salud: informe de la Conferencia Internacional sobre Atención Primaria de Salud, Declaración de Alma-Ata. Ginebra; 1978. (Serie Salud para Todos (OMS), 1).

12. Shepard RJ. Physical activity, fitness and health: the current consensus. Quest. 1995;47(3):228-303.

13. Siqueira FV, Facchini LA, Piccini RX, Tomasi E, Thumé E, Silveira DS, et al. Atividade Física em adultos e idosos residentes em áreas de abrangência de unidades básicas de saúde de municípios das regiões Sul e Nordeste do Brasil. Cad Saude Publica. 2008;24(1):39-54. DOI:10.1590/S0102$311 \times 2008000100005$

14. Spencer EH, Frank E, Elon LK, Hertzberg VS, Serdula MK, Galuska DA. Predictors of nutrition counseling behaviors and attitudes in US medical students. Am J Clin Nutr. 2006;84(3):655-62. 